\title{
Endotracheal calcineurin inhibition ameliorates injury in an experimental model of lung ischemia-reperfusion
}

\author{
Steven M. Woolley, MRCS \\ Alexander S. Farivar, MD \\ Babu V. Naidu, FRCS \\ Matthew Rosengart, MD \\ Robert Thomas, BA \\ Charles Fraga, MS \\ Michael S. Mulligan, MD, FACS
}

From the Division of Cardiothoracic Surgery, University of Washington, Seattle, Wash.

Read at the Eighty-third Annual Meeting of The American Association for Thoracic Surgery, Boston, Mass, May 4-7, 2003.

Received for publication May 2, 2003; revisions requested Sept 25, 2003; accepted for publication Sept 30, 2003

Address for reprints: Michael Mulligan, MD, FACS, Box 356310, University of Washington, 1959 NE Pacific Street, Seattle, WA 98195 (E-mail: msmmd@u. washington.edu).

J Thorac Cardiovasc Surg 2004;127:376-84 $0022-5223 / \$ 30.00$

Copyright ( $\odot 2004$ by The American Association for Thoracic Surgery

doi:10.1016/j.jtcvs.2003.09.034
Objectives: We previously demonstrated that calcineurin inhibitors given intravenously ameliorate experimental lung ischemia-reperfusion injury. This study evaluates whether these effects can be achieved when these agents are delivered endotracheally.

Methods: Left lungs of Long Evans rats were rendered ischemic for 90 minutes and reperfused for up to 4 hours. Treated animals received tacrolimus endotracheally at doses of $0.2,0.1$, or $0.025 \mathrm{mg} / \mathrm{kg} 60$ minutes before ischemia. Injury was quantitated in terms of vascular permeability. Additional animals treated at a dose of $0.1 \mathrm{mg} / \mathrm{kg}$ were assessed for lung tissue myeloperoxidase content and bronchoalveolar lavage leukocyte content. Bronchoalveolar lavage fluid was assessed for cytokine and chemokine content by enzyme-linked immunosorbent assay. Tissue samples were processed for nuclear factor- $\kappa \mathrm{B}$ activation, and blood levels of tacrolimus were measured in treated animals.

Results: Left lung vascular permeability was reduced in treated animals in a dosedependent fashion compared with controls. The protective effects correlated with a $47 \%$ $(0.50 \% \pm 0.06 \%$ vs $0.27 \% \pm 0.08 \%$, respectively) reduction in tissue myeloperoxidase content $(P<.004)$ and marked reductions in bronchoalveolar lavage leukocyte accumulation. This protection was also associated with decreased nuclear factor- $\kappa \mathrm{B}$ activation and diminished expression of proinflammatory mediators. Blood tacrolimus levels in treated animals at 4 hours of reperfusion were undetectable.

Conclusions: Tacrolimus administered endotracheally is protective against lung ischemia-reperfusion injury in our model. This protection is associated with a decrease in nuclear factor- $\kappa \mathrm{B}$ activation. This route of tacrolimus administration broadens its potential clinical use and decreases concerns about systemic and renal toxicity. It may be a useful therapy in lung donors to protect against lung ischemiareperfusion injury.

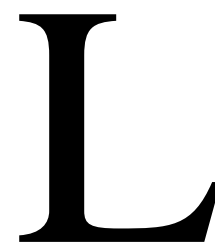

ung ischemia-reperfusion injury (LIRI) is a major complication after lung transplantation and is associated with an increase in vascular permeability that manifests itself clinically as pulmonary edema. ${ }^{1}$ Despite improvements in organ preservation techniques, ${ }^{2}$ LIRI persists and compromises both early and late graft function. In animal models, this injury has been shown to be biphasic. ${ }^{3}$ The late phase is dependent on neutrophil recruitment, whereas the early stage is independent of it, occurring within 15 minutes of reperfusion. The early phase also seems to involve signaling events that lead to the full development of tissue injury after several hours of reperfusion. 
Tacrolimus (TAC) is an immunosuppressive agent that has been used in solid-organ transplantation for almost 2 decades. The immunomodulatory effects of TAC involve the inhibition of the tyrosine phosphatase calcineurin. TAC is a macrolide antibiotic that binds to a cytoplasmic protein, FK binding protein, and inhibits calcineurin. TAC is 100 times more potent than the other commonly used calcineurin inhibitor, cyclosporine A (CsA). Inhibition of calcineurin in turn reduces the expression of a number of transcription factors, including nuclear factor of activated $\mathrm{T}$ cell (NF-AT), Oct-1, AT-1, and NF- $\kappa$ B. All of these transcription factors can bind to different sequences on the interleukin-2 promoter. The most critical of these in immunomodulation is NF-AT. Decreased transcription factor activity results in a diminished production of interleukin-2 messenger RNA and protein, which subsequently blunts the T-cell response that mediates cellular rejection.

Although effects on NF-AT are known to be central to the immunomodulatory effects of TAC, its effects on NF- $\kappa \mathrm{B}$ are of great interest in inflammatory conditions. $\mathrm{NF}-\kappa \mathrm{B}$ is a transcription factor that has a wide range of activities including modulation of cell growth and death, regulation of immune function, and apparent effects on acute reperfusion injury. ${ }^{4}$ Calcineurin inhibitors have been shown to be protective in reperfusion injury of the brain, kidney, liver, and heart. ${ }^{5-8}$ We previously demonstrated that intravenous calcineurin inhibition with both CsA and TAC is effective in ameliorating experimental LIRI. ${ }^{9}$ We also showed that CsA attenuates proinflammatory chemokine secretion in alveolar macrophages in an in vitro hypoxia and reoxygenation model. ${ }^{10}$ Previous in vitro studies delineated the mechanism by which CsA and TAC may inhibit NF- $\kappa \mathrm{B}$ activation. ${ }^{11,12}$ It has been suggested that calcineurin inhibition blocks the degradation of $\mathrm{I} \kappa \mathrm{B}$, the cytoplasmic protein that binds NF- $\kappa \mathrm{B}$ in its inactive form, therefore decreasing the levels of active NF- $\kappa$ B. In addition, calcineurin inhibition decreases processing of proform precursors of $\mathrm{NF}-\kappa \mathrm{B}$ to its functional heterodimeric form. Therefore, calcineurin inhibitors lead to diminished concentration of active NF- $\kappa \mathrm{B}$ in the nucleus, which leads to decreased production of mRNA for proinflammatory cytokines, neutrophil adhesion molecules, procoagulant proteins, and vasoconstrictive substances. It seems that many of the antiinflammatory properties of calcineurin inhibitors are modulated through its effects on NF- $\kappa \mathrm{B}$ and are separate from its immunomodulation, which occurs through NF-AT inhibition. Increased sensitivities among macrophages and neutrophils, as opposed to $\mathrm{T}$ lymphocytes, help explain the differential anti-inflammatory and immunomodulatory effects of the same drugs.

Early studies of calcineurin inhibition during organ ischemia-reperfusion (I/R) injury involved animals that were treated with either CsA or TAC during the week preceding the experiment. ${ }^{13-15}$ This makes clinical translation difficult. Although protection was seen in these models, such a strategy would be very difficult to implement in human transplant recipients. More recent studies in cardiac and cerebral models have shown protection against I/R injury when administered at or close to the time of onset of ischemia or even with treatment early after its onset. ${ }^{16,17}$

We previously demonstrated that the alveolar macrophage is the central orchestrating cell in LIRI. ${ }^{18,19}$ Endotracheal agents targeting these cells have been protective in other models of acute lung injury. ${ }^{20}$ Therefore, we hypothesized that delivering TAC endotracheally would treat these cells directly and provide protection similar to that seen with intravenous administration. We further hypothesized that systemic levels of TAC would be low with this route of delivery and that this could be useful in reducing potential negative side effects of intravenous drug administration. Finally, knowing that previous studies have suggested that the protective effects of TAC may be mediated by modulation of NF- $\kappa \mathrm{B}$, we hypothesized that any protection would be associated with reduced activation of NF- $\kappa \mathrm{B}$ and expression of proinflammatory mediators.

\section{Methods \\ Reagents}

All reagents were purchased from Sigma Chemical (St. Louis, Mo) unless otherwise specified. The commercial preparation of the calcineurin inhibitor TAC (Fujisawa, Deerfield, Ill) was obtained from the inpatient pharmacy at the University of Washington in Seattle.

\section{Animal Model}

Pathogen-free, adult male Long-Evans rats (Simonsen Labs, Gilroy, Calif), weighing 250 to $275 \mathrm{~g}$, were used for all experiments. All animals received humane care in compliance with the "Guide for the Care and Use of Laboratory Animals," published by the National Society for Medical Research and the National Institute of Health. An in situ warm I/R model was used as described previously. ${ }^{9}$ Treated animals received TAC through their endotracheal tube at doses of $0.2,0.1$, or $0.025 \mathrm{mg} / \mathrm{kg} 1$ hour before any experimental procedure. Further animals received an equivalent volume of vehicle. In brief, anesthesia was induced with pentobarbital, and animals were ventilated by tracheostomy (Harvard Rodent Ventilator, Harvard Apparatus Inc, Holliston, Mass). All animals received $0.2 \mathrm{mg}$ of atropine intramuscularly and 50 units of intravenous heparin. Anesthesia was maintained with inhaled halothane. The left lung was mobilized atraumatically through an anterolateral thoracotomy, and the left pulmonary artery, veins, and main stem bronchus were occluded with a noncrushing microvascular clamp. At the end of the 90-minute ischemic period, the clamp was removed and the lung was ventilated and reperfused for periods of up to 4 hours. At the conclusion of the reperfusion period, blood samples were obtained from the inferior vena cava, the heart lung block was rapidly excised, and the pulmonary circulation was flushed with $20 \mathrm{~mL}$ of normal saline through the main pulmonary artery. 


\section{Lung Permeability Index}

To quantitate lung injury, a permeability index was determined as described previously. ${ }^{9,18} \mathrm{I}^{125}$-radiolabeled bovine serum albumin (NEN Life Sciences, Boston, Mass), titrated to an activity of 800,000 counts $\cdot \min \cdot$ dose, was intravenously injected in a final volume of $1 \mathrm{~mL}$ of $1 \%$ bovine serum albumin phosphate-buffered saline solution 5 minutes before removal of the hilar clamp. At the end of the experiment, the radioactivity counts were quantitated in the left lung and in $1 \mathrm{~mL}$ of inferior vena cava blood using a scintillation counter. The permeability index was then calculated as follows:

$$
\text { Permeability index }=\frac{\text { Left lung }(\text { counts } / \mathrm{min})}{1.0 \mathrm{~mL} \text { blood }(\text { counts } / \mathrm{min})}
$$

This ratio corrected for any variation in systemic blood levels of radioactivity and provided a reproducible measure of lung microvascular permeability.

\section{Myeloperoxidase Assay}

Tissue myeloperoxidase (MPO) content was used to quantitate neutrophil accumulation in the left lung as described previously. ${ }^{9}$ Lung samples were homogenized in a phosphate buffer containing $0.5 \%$ hexadecyltrimethylammonium bromide. Samples were then assayed for the ability to decompose $\mathrm{H}_{2} \mathrm{O}_{2}$ in the presence of $\mathrm{O}$-dianisidine dihydrochloride by the change in absorption at 460 nm during 1 minute.

\section{Bronchoalveolar Lavage}

Additional animals underwent bronchoalveolar lavage (BAL) at the time of death. Through the tracheostomy, lungs were lavaged individually by clamping the contralateral hilum and instilling 3.0 $\mathrm{mL}$ of saline that was flushed and aspirated 3 times. This fluid was centrifuged $\left(1500 \mathrm{~g} \times 8\right.$ minutes at $\left.4^{\circ} \mathrm{C}\right)$ to pellet the cells, and the supernatant was snap-frozen for subsequent cytokine analysis after the addition of a protease cocktail inhibitor (leupeptin $1 \mu \mathrm{g} / \mathrm{mL}$, aprotinin $1 \mu \mathrm{g} / \mathrm{mL}$, trypsin inhibitor $5 \mu \mathrm{g} / \mathrm{mL}$, and pepstatin A 1 $\mu \mathrm{g} / \mathrm{mL}$ ). The red blood cells were lysed, and the pellet was resuspended in normal saline. Total nucleated cells were counted using a hemacytometer (Hausser Scientific, Reading, Pa).

\section{Enzyme-Linked Immunosorbent Assay of BAL for Cytokine Content}

Sandwich enzyme-linked immunosorbent assays for macrophage inflammatory protein (MIP)- $1 \alpha$, cytokine-induced neutrophil chemoattractant (CINC), and tumor necrosis factor (TNF)- $\alpha$ were developed in our laboratory. ${ }^{18}$ Fifty microliters of a $1-\mu \mathrm{g} / \mathrm{mL}$ protein-A purified specific antichemokine antibody (Peprotech, Rocky Hills, NJ) in a carbonate-coating buffer solution ( $\mathrm{pH} 9.6$ ) was added to a 96-well (Dynex, Chantilly, Va) immunoassay plate. The plate was incubated overnight at $4{ }^{\circ} \mathrm{C}$ and washed with phosphate-buffered saline with $0.05 \%$ Tween. One percent bovine serum albumin in saline was used to block nonspecific binding (30-minute incubation at $37^{\circ} \mathrm{C}$ ). Samples and standards were diluted in saline, and $50 \mu \mathrm{L}$ was added to each well (1-hour incubation at $37^{\circ} \mathrm{C}$ ). A secondary, affinity-purified, biotinylated antibody (Peprotech, Rocky Hills, NJ) specific to the epitope being studied $(0.5-2 \mu \mathrm{g} / \mathrm{mL}$ ) was added to each well (1-hour incubation at $37^{\circ} \mathrm{C}$ ). After a 30 -minute incubation with a streptavidin-horseradish-peroxidase conjugate (Pierce, Rockford, Ill), the assay was developed by adding O-phenylenediamine dihydrochloride substrate. The linear sensitivity range of the assays was determined, and the assays showed no cross-reactivity with each other. Samples and standards were run in triplicate, and well-to-well variation did not exceed $5 \%$.

\section{Electrophoretic Mobility Shift Assay}

Left lungs were snap-frozen at the end of the experimental protocols. These frozen tissue samples were ground to a fine powder and then suspended in $4.0 \mathrm{~mL}$ of buffer containing $0.06 \%$ Nonidet $\mathrm{P}-40,150 \mathrm{mmol} / \mathrm{L} \mathrm{NaCl}, 10 \mathrm{mmol} / \mathrm{L}$ HEPES, $1 \mathrm{mmol} / \mathrm{L}$ EDTA, and $0.5 \mathrm{mmol} / \mathrm{L}$ PMSF. The solution was then homogenized and centrifuged for 15 seconds $(12,000 \mathrm{~g})$. The pellet was discarded, and the supernatant was cooled to $4^{\circ} \mathrm{C}$. The supernatant was then centrifuged again for 15 seconds $(12,000 \mathrm{~g})$. The resultant pellet was suspended in $40 \mu \mathrm{L}$ of buffer containing $40 \mathrm{mmol} / \mathrm{L} \mathrm{NaCl}, 20$ $\mathrm{mmol} / \mathrm{L}$ HEPES, $0.2 \mathrm{mmol} / \mathrm{L}$ EDTA, $1.2 \mathrm{mmol} / \mathrm{L} \mathrm{MgCl}_{2}, 0.5$ $\mathrm{mmol} / \mathrm{L}$ PMSF, $0.5 \mathrm{mmol} / \mathrm{L}$ DDT, $25 \%$ glycerol, $5 \mu \mathrm{g} / \mathrm{mL}$ aprotinin, and $5 \mu \mathrm{g} / \mathrm{mL}$ leupeptin for 20 minutes at $4{ }^{\circ} \mathrm{C}$. This solution was then centrifuged for 5 minutes, the pellet was discarded, and the supernatant containing the nuclear protein was stored at $-70^{\circ} \mathrm{C}$. Quantification of nuclear protein was performed using the bichinoic acid assay.

Ten micrograms of nuclear protein were incubated in a binding reaction with either double-stranded $32 \mathrm{P}$ end-labeled oligonucleotide containing the NF- $\kappa \mathrm{B}$ or activator protein- 1 consensus binding sequence (Promega, Madison, Wis). The binding reaction was performed at room temperature for 60 minutes, and the proteins were resolved on a $6 \%$ nondenaturing polyacrylamide gel at $100 \mathrm{~V}$ for 1 to 2 hours. The gels were dried and autoradiographed. Densitometry was performed with Image $\mathrm{J}$ software (version 1.2; Cybernetics Corp, Silver Spring, Md) to assess relative signal intensity.

\section{Blood TAC Levels}

Blood levels of TAC were measured in animals treated at a dose of $0.1 \mathrm{mg} / \mathrm{kg} 1$ hour after administration of the drug and at 4 hours of reperfusion (6.5 hours postadministration). All measurements were performed by the University of Washington clinical laboratories.

\section{Statistical Analysis}

All data are presented as mean \pm SEM. Data sets were analyzed using Microsoft Excel 2000 and SPSS (version 10, Microsoft Inc, Redmond, Wash). Comparisons between control groups or between positive controls and treated animals were performed using the Student $t$ test. Analyses between more than 2 groups were performed with analysis of variance with control for multiple comparisons.

\section{Results}

\section{Effect of Endotracheal TAC Pretreatment on Lung Vascular Permeability}

The left lung permeability index increased from $0.09 \pm$ 0.005 in unmanipulated animals to $0.80 \pm 0.04$ among animals receiving vehicle and undergoing 90 minutes of is- 
chemia followed by 4 hours of reperfusion $(P<.001)$. Endotracheal TAC pretreatment at doses of $0.2,0.1$, and $0.025 \mathrm{mg} / \mathrm{kg}$ reduced lung vascular permeability by $70 \%$, $67 \%$, and $30 \%$, respectively, after 4 hours of reperfusion (Figure 1) $(P<.005$ for all 3 groups, $\mathrm{N}=4)$. Because no statistically significant difference was seen between protection with doses of 0.2 and $0.1 \mathrm{mg} / \mathrm{kg}$ of TAC, the lower dose of $0.1 \mathrm{mg} / \mathrm{kg}$ was chosen for all further experiments.

\section{Effect of Endotracheal TAC Pretreatment on Lung MPO Content}

Lung tissue MPO content increased from $0.012 \pm 0.003$ among unmanipulated animals to $0.51 \pm 0.3$ in the vehicletreated group undergoing 90 minutes of ischemia followed by 4 hours of reperfusion $(P<.001)$. There was a $48 \%$ reduction in tissue MPO content among TAC-treated animals undergoing the same periods of $\mathrm{I} / \mathrm{R}$ at a dose of 0.1 $\mathrm{mg} / \mathrm{kg}(0.27 \pm 0.04, P<.004, \mathrm{~N}=4)$ (Figure 2).

\section{Effect of Endotracheal TAC Pretreatment on BAL Cell Counts}

BAL cell count increased 20 -fold among vehicle-treated animals undergoing 90 minutes of ischemia followed by 4 hours of reperfusion compared with unmanipulated animals $(0.84 \pm 0.13-16.5 \pm 0.81, P<.001)$. There was a $77 \%$ reduction in BAL cell count associated with TAC $(0.1$ $\mathrm{mg} / \mathrm{kg}$ ) treatment in animals undergoing the same periods of $\mathrm{I} / \mathrm{R}(4.38 \pm 0.4, P<.001, \mathrm{~N}=4)$ (Figure 3$)$.

\section{Effect on Cytokine Content in BAL Associated with Endotracheal TAC Pretreatment}

Three groups were studied: unmanipulated animals, vehicle-treated animals that underwent 90 minutes of ischemia followed by 4 hours of reperfusion, and endotracheal TACpretreated animals $(0.1 \mathrm{mg} / \mathrm{kg})$ subjected to the same LIRI protocol. CINC expression increased from $0.052 \pm 0.003$ to $2.9 \pm 0.2 \mathrm{ng} / \mathrm{mL}$ between unmanipulated and vehicletreated animals. A 65\% decrease was demonstrated in the TAC-treated group to $1.04 \pm 0.1 \mathrm{ng} / \mathrm{mL}(P<.002)$ (Figure $4, A)$. MIP- $1 \alpha$ increased from $0.03 \pm 0.002 \mathrm{ng} / \mathrm{mL}$ to 0.34 $\pm 0.019 \mathrm{ng} / \mathrm{mL}$ between unmanipulated and vehicle-treated animals, respectively. This increase was almost completely blocked with TAC treatment, giving a reduction of $93 \%$ to $0.055 \pm 0.004 \mathrm{ng} / \mathrm{mL}(P<.001)$ (Figure $4, B)$. TNF- $\alpha$ content of lavage fluid was increased in vehicle-treated animals undergoing 90 minutes of ischemia and 4 hours of reperfusion when compared with unmanipulated animals $(27.5 \pm 6.8 \mathrm{pg} / \mathrm{mL}$ vs $67.5 \pm 4.4 \mathrm{pg} / \mathrm{mL})$. This increase was decreased by $69 \%$ with TAC treatment to $39.6 \pm 4.2 \mathrm{pg} / \mathrm{mL}$ $(P<.03$ ) (Figure 4, $C$ ).

\section{Effect on NF- $\kappa$ B Activation by Endotracheal TAC Pretreatment}

To explore the potential mechanisms mediating the protection seen with TAC in LIRI, the effects on expression of the

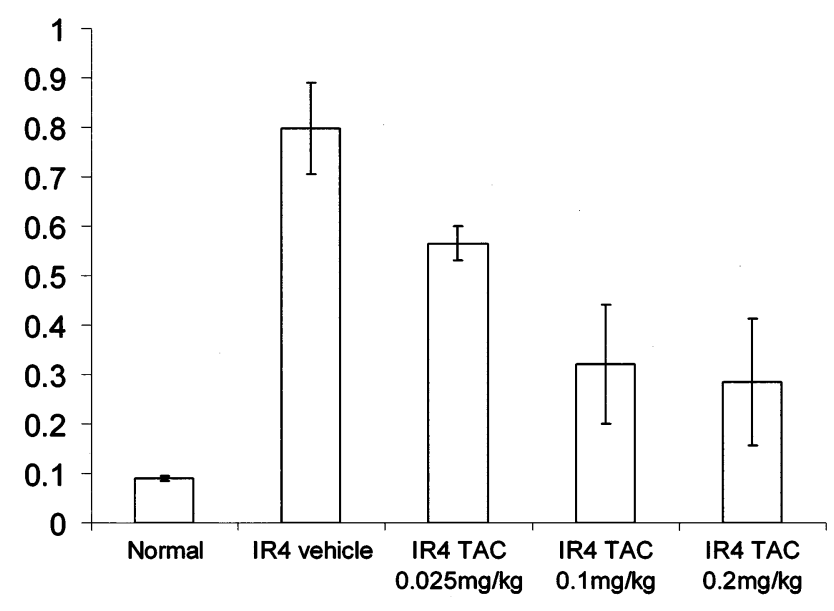

Figure 1. There is a marked increase in permeability index between unmanipulated animals (normal) and vehicle-treated animals undergoing ischemia of $\mathbf{9 0}$ minutes followed by reperfusion of 4 hours (IR4). TAC-treated animals showed protection against increased vascular permeability at all 3 doses studied $(P<.005$ for all 3 groups). There was no significant difference in permeability index between the 0.2 and $0.1 \mathrm{mg} / \mathrm{kg}$ doses. TAC, Tacrolimus; $I / R$, ischemia-reperfusion.

proinflammatory transcription factor NF- $\kappa \mathrm{B}$ were assessed. The band that is representative for NF- $\kappa \mathrm{B}$ was specified using cold competition with nonlabeled oligonucleotide. The time point of 15 minutes of reperfusion was used because this is the early peak in injury, and we previously demonstrated high levels of NF- $\kappa$ B activation at this time in our model when compared with unmanipulated animals (data not shown). A representative electrophoretic mobility shift assay (EMSA) is shown in Figure 5, A. As can be seen, there is marked NF- $\kappa \mathrm{B}$ nuclear translocation in vehicletreated animals undergoing 90 minutes of ischemia followed by 15 minutes of reperfusion, and this is decreased in endotracheal TAC-treated lungs $(\mathrm{N}=3)$. Densitometric analysis is depicted below the autoradiograph and reveals a statistically significant reduction in NF- $\kappa \mathrm{B}$ nuclear translocation with TAC treatment $(P<.003)$ (Figure 5, B).

\section{Blood TAC Levels}

Blood TAC levels in animals receiving the $0.1 \mathrm{mg} / \mathrm{kg}$ dose averaged $5.0 \pm 1.0 \mathrm{ng} / \mathrm{mL} 1$ hour after administration. At 4 hours of reperfusion, blood TAC levels were below detectable limits in all animals studied at this dose.

\section{Discussion}

Calcineurin inhibitors such as TAC and CsA have been shown to provide marked protection against I/R injury of several organs, including the brain, heart, kidney, and liv- 


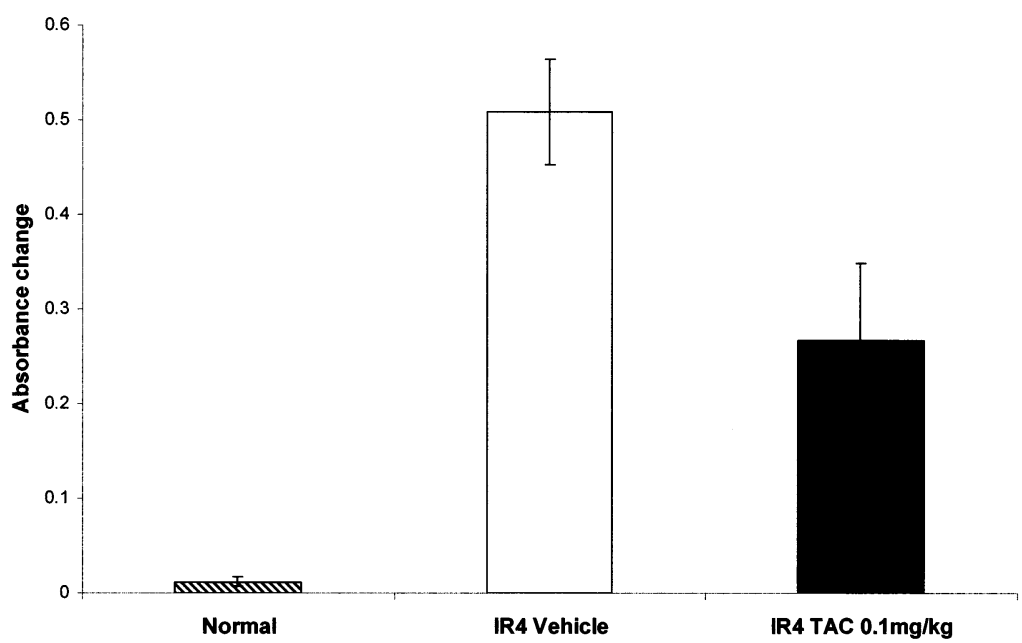

Figure 2. Left lung tissue MPO content increased dramatically between unmanipulated animals (normal) and vehicle-treated animals undergoing $\mathbf{9 0}$ minutes of ischemia followed by $\mathbf{4}$ hours of reperfusion (IR4). MPO content was significantly decreased with TAC treatment $(0.1 \mathrm{mg} / \mathrm{kg})(P<.001)$.

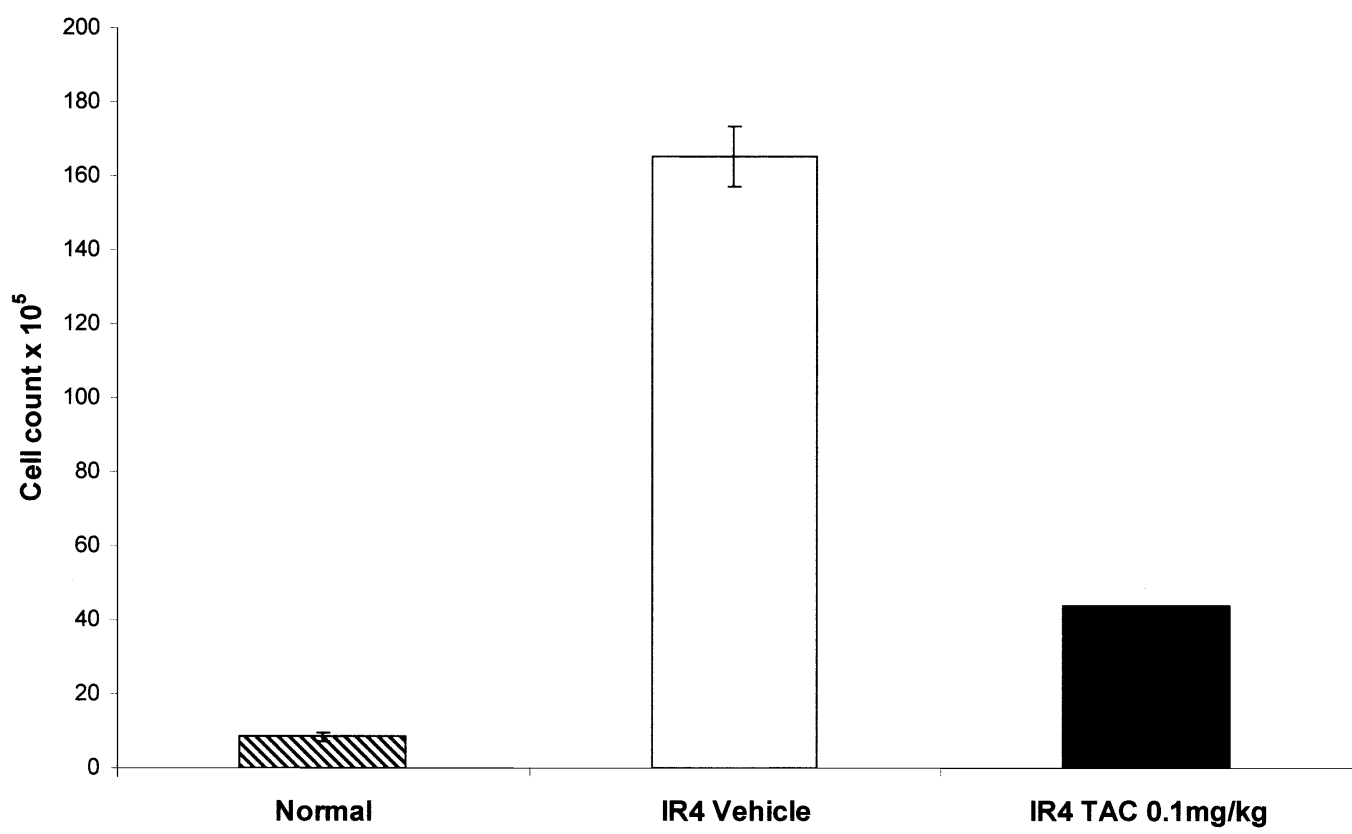

Figure 3. BAL cell count increased 20-fold between unmanipulated animals (normal) and vehicle-treated animals undergoing 90 minutes of ischemia followed by 4 hours of reperfusion (IR4). Cell counts were decreased by $77 \%$ in the TAC-treated group $(0.1 \mathrm{mg} / \mathrm{kg})(P<.001)$.

er. $^{5-8}$ There are several mechanisms that have been suggested for this protection, which are in 3 broad categories: effects on mitochondrial stabilization, effects on microcirculation, and effects on transcriptional regulation. ${ }^{21}$ Most of the work studying the effects on mitochondrial stabilization has been performed in tissues rich in mitochondria, such as the heart and liver. In I/R injury in such tissues, CsA and TAC have been shown to abrogate calcium influx and decrease mitochondrial transition pore opening, which leads to decreased tissue injury. ${ }^{22,23}$ However, these mitochondrial effects seem to play a lesser role in lung tissue. Abnormalities in tissue microcirculation are known to be important in I/R injury. It has been reported that protective effects of CsA and TAC in hepatic I/R are associated with maintenance or rapid restoration of normal parenchymal blood flow. ${ }^{13,22}$ Endothelin-1 expression and effects on ni- 

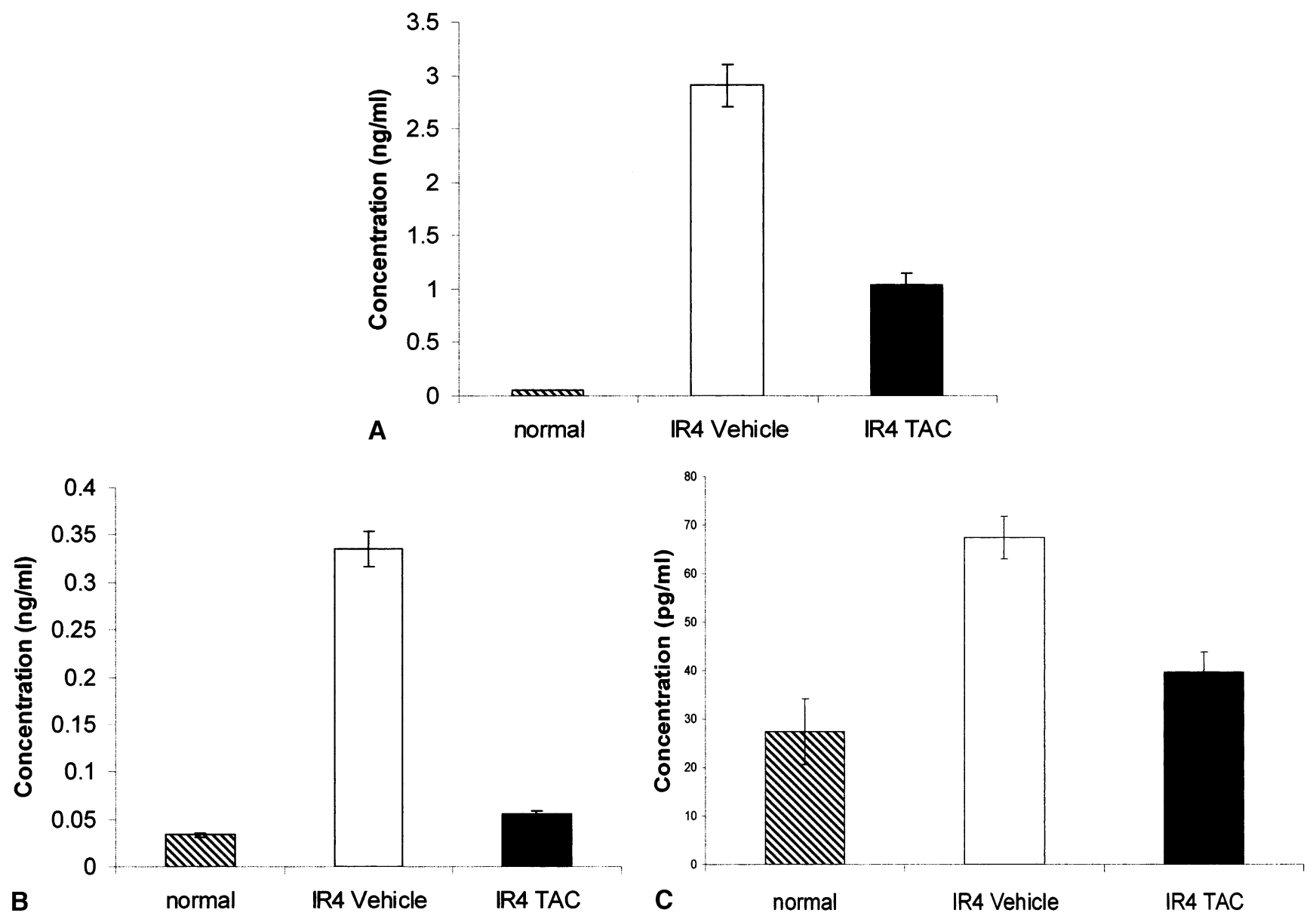

Figure 4. A, There was a marked increase in BAL CINC content in vehicle-treated animals undergoing 90 minutes of ischemia followed by 4 hours of reperfusion (IR4) compared with unmanipulated animals (normal). TAC treatment $(0.1 \mathrm{mg} / \mathrm{kg})$ significantly decreased CINC levels in BAL fluid $(P<.002)$. B, MIP-1 $\alpha$ content of BAL fluid was increased in vehicle-treated animals undergoing 90 minutes of ischemia followed by 4 hours of reperfusion (IR4) compared with unmanipulated animals (normal). TAC treatment $(0.1 \mathrm{mg} / \mathrm{kg})$ decreased this by $93 \%(P<.001)$. C, TNF- $\alpha$ content in BAL increased in vehicle-treated animals undergoing 90 minutes of ischemia followed by 4 hours of reperfusion (IR4) compared with unmanipulated animals (normal). TAC treatment $(0.1 \mathrm{mg} / \mathrm{kg})$ decreased this by $69 \%(P<.03)$.

tric oxide production have been shown to mediate variations in vasomotor tone. In many organs, postischemic vasoconstriction is believed to decrease tissue edema through decreased starling forces. However, such vasoconstriction in the lung may adversely affect lung function and proper gas exchange.

In LIRI, the dominant mechanism of protection by calcineurin inhibition likely relates to effects on transcriptional up-regulation of proinflammatory mediators. We previously demonstrated that intravenous treatment with both CsA and TAC was protective in a rat model of LIRI. ${ }^{9}$ Furthermore, this protection was shown to be associated with modulation of the transcription factor NF- $\kappa \mathrm{B}$ and a decrease in proinflammatory mediator expression. Recent work in renal $\mathrm{I} / \mathrm{R}$ injury has demonstrated changes in mitogen-activated protein kinase activity with both CsA and TAC treatment. ${ }^{6}$ These effects on upstream signaling kinases may explain further the protective effects of calcineurin inhibition in $I / R$ injury.

The alveolar macrophage has been identified as a key effector cell in lung reperfusion injury. ${ }^{24}$ We have previously shown that depletion of macrophages or inhibition of macrophage function are both protective in LIRI. ${ }^{18,19}$ It seems that alveolar macrophages play a very important role in the early stages of reperfusion injury and set up an inflammatory cascade leading to the later full blown injury. In previous in vitro studies, we showed that CsA attenuates the expression of a number of proinflammatory mediators 


\section{Cold IR15 IR15 IR15 TAC TAC TAC}

A
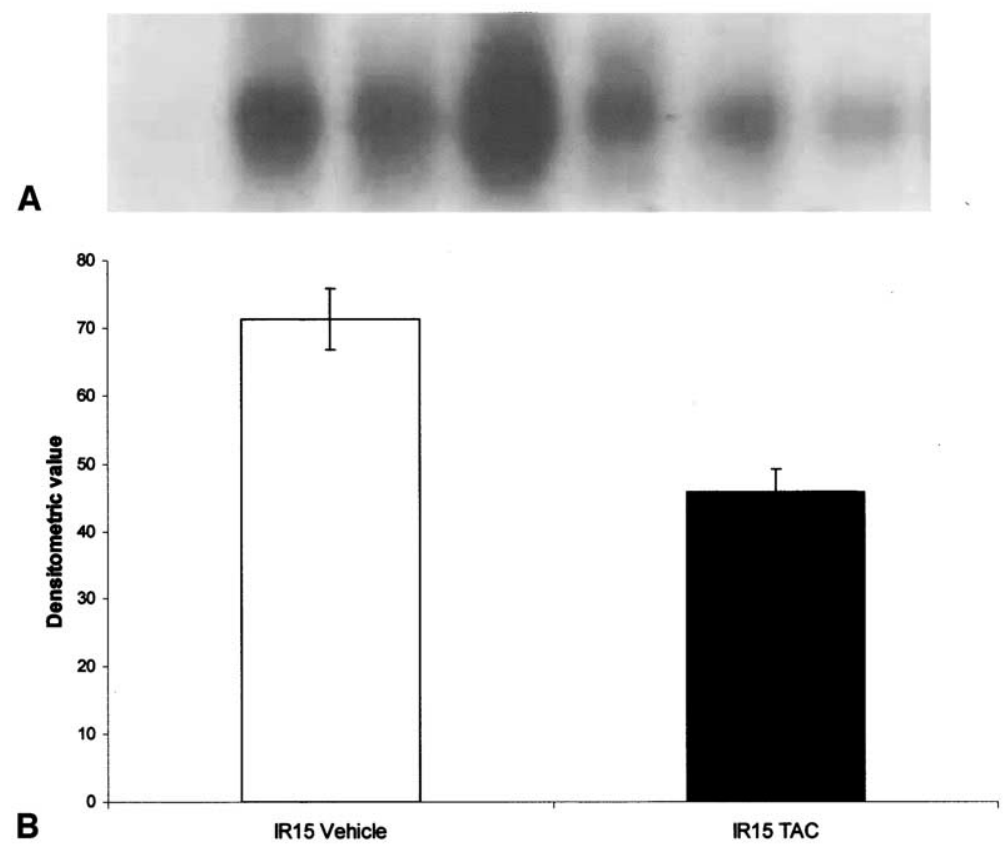

Figure 5. A, Representative EMSA for NF- $\kappa$ B nuclear translocation. Cold competition (cold lane); vehicle-treated animals that underwent $\mathbf{9 0}$ minutes of ischemia followed by $\mathbf{1 5}$ minutes of reperfusion (IR15 lanes); TAC-treated animals $(0.1 \mathrm{mg} / \mathrm{kg}$ undergoing the same periods of $\mathrm{I} / \mathrm{R}$ (TAC lanes). There is less activation of NF- $\kappa B$ in the TAC-treated animals than in the vehicle-treated animals. B, Relative densitometry from EMSAs for NF- $\kappa$ B nuclear translocation. There is a decrease in relative densitometric value with TAC treatment when compared with the vehicle-treated groups $(P<.003)$

secreted by primary harvested alveolar macrophages in response to hypoxia followed by reoxygenation. ${ }^{10}$ Therefore, by delivering a drug into the intra-alveolar compartment through the endotracheal route, these orchestrating cells would be treated directly, limiting their early response (which incites the inflammatory cascade leading to LIRI). The endotracheal route would also likely allow protection to be achieved with a lower systemic drug concentration because the drug is being delivered directly to the cells involved in LIRI rather than systemically. This route would therefore be more attractive in the clinical setting of organ donors because the potential problems of renal or hepatic toxicity would be minimal.

In this study, we showed that TAC is protective against LIRI when delivered through the endotracheal route. Significant protection against an increase in lung vascular permeability was achieved at all 3 doses used. Furthermore, this protection was not significantly different between the 0.2 and $0.1 \mathrm{mg} / \mathrm{kg}$ doses. The degree of protection achieved at both of these doses was greater than that previously demonstrated with intravenous TAC administration at a dose of $0.2 \mathrm{mg} / \mathrm{kg}^{9}{ }^{9}$ This protection was associated with a decrease in both lung tissue and alveolar space neutrophil sequestration as assessed by MPO content and BAL cell counts. Endotracheal TAC pretreatment was associated with a decrease in expression of the proinflammatory cytokines CINC, TNF- $\alpha$, and MIP- $1 \alpha$, as well as a decrease in nuclear translocation of NF- $\kappa$ B. This is in keeping with our and other investigators' previous work showing that calcineurin inhibition modulates NF- $\kappa$ B activity. ${ }^{8,9}$ The results of TAC blood levels were very encouraging: At the dose used for most experiments $(0.1 \mathrm{mg} / \mathrm{kg})$, TAC blood levels were below detectable limits at 4 hours of reperfusion. Earlier on at 1 hour after administration, the mean TAC blood level was $5.0 \mathrm{ng} / \mathrm{mL}$, which is still at the lower end of the normal therapeutic range used for immunosuppression protocols with TAC. Furthermore, in such protocols, the TAC blood levels used to establish dosing are trough levels normally measured 12 hours after the last dose. It is these trough levels that are important in determining TAC toxicity. Our 1-hour postadministration time point would be unlikely to represent a trough level, whereas our 4-hour reperfusion time point would be nearer to a true trough level. Therefore, because the blood levels at 4 hours of reperfusion (6.5 hours after administration) are undetectable, it is unlikely that this dose of TAC would lead to toxic effects. 


\section{Conclusion}

We have demonstrated that endotracheal TAC administration is protective against LIRI in our experimental model. Furthermore, this protection is associated with a decrease in both NF- $\kappa \mathrm{B}$ activity and proinflammatory cytokine expression. This represents a potentially useful therapy in lung transplantation; however, further work is needed to evaluate its effects in a whole-lung transplant model. One of the potential concerns is that inhibition of NF- $\kappa \mathrm{B}$ in transplanted lungs is likely to have effects on several processes as well as LIRI. For example, there is the potential that prolonged NF- $\kappa \mathrm{B}$ inhibition could lead to delayed healing. We believe that if TAC is administered as a single dose into the lungs before ischemia and during the early reperfusion period, the likelihood of such complications is low. We are planning to investigate this in future experiments using a whole-lung transplant model.

\section{References}

1. Meyers BF, Lynch J, Trulock EP, Guthrie TJ, Cooper JD, Patterson GA. Lung transplantation: a decade of experience. Ann Surg. 1999; 230:362-70.

2. Wittwer T, Wahler T, Fehrenbach A, Elki S, Haverich A. Improvement of pulmonary preservation with Celsior and Perfadex: impact of storage time on early post-ischemic lung function. J Heart Lung Transplant. 1999;18:1198-201.

3. Eppinger MJ, Jones ML, Deeb GM, Bolling SF, Ward PA. Pattern of injury and the role of neutrophils in reperfusion injury of rat lung. J Surg Res. 1995;58:713-8.

4. Baeuerle PA, Baltimore D. NF- $\kappa$ B: ten years later. Cell. 1996;87:1320.

5. Giordani F, Benetolli A, Favero-Filho LA, Lima KC, Cestari Junior L, Milani H. Tacrolimus (FK506) reduces ischemia-induced hippocampal damage in rats: a 7- and 30-day study. Braz J Med Biol Res. 2003;36: 495-502.

6. Yang CW, Ahn HJ, Han HJ, Kim WY, Li C, Shin MJ, et al. Pharmacological preconditioning with low-dose cyclosporine or FK506 reduces subsequent ischemia/reperfusion injury in rat kidney. Transplantation. 2001;72:1753-9.

7. St Peter SD, Post DJ, Rodriguez-Davalos MI, Douglas DD, Moss AA, Mulligan DC. Tacrolimus as a liver flush solution to ameliorate the effects of ischemia/reperfusion injury following liver transplantation. Liver Transpl. 2003;9:144-9.

8. Squadrito F, Altavillla D, Squadrito G, Saitta A, Deodat B, Arlotta M, et al. Tacrolimus limits polymorphonuclear accumulation and protects against myocardial ischemia-reperfusion injury. $\mathrm{J} \mathrm{Mol} \mathrm{Cell} \mathrm{Cardiol.}$ 2000;32:429-40.

9. Krishnadasan B, Naidu B, Rosengart M, Barnes A, Verrier ED, Mulligan MS, et al. Decreased lung ischemia-reperfusion injury in rats after preoperative administration of cyclosporine and tacrolimus. J Thorac Cardiovasc Surg. 2002;123:756-67.

10. Naidu BV, Krishnadasan B, Byrne K, Rosengart M, Verrier ED, Mulligan MS, et al. Regulation of chemokine expression by cyclosporine A in alveolar macrophages exposed to hypoxia and reoxygenation. Ann Thorac Surg. 2002;74:899-905.

11. Meyer S, Kohler G, Joly A. Cyclosporine A is an uncompetitive inhibitor of proteasome activity and prevents NF- $\kappa \mathrm{B}$ activation. FEBS Lett. 1997;413:354-8.

12. Hughes K, Antonsson A, Grundstrom T. Calmodulin dependence of NfkappaB activation. FEBS Lett. 1998;44:132-6.

13. Travis DL, Fabia R, Netto GG, Husberg BS, Goldstein RM, Klintman $\mathrm{GB}$, et al. Protection by cyclosporine A against normothermic liver ischemia-reperfusion in pigs. $J$ Surg Res. 1998;75:116-26.

14. Dhar DK, Takemoto YI, Nagasue N, Uchida M, Ono T, Nakamura T.
FK-506 maintains cellular calcium homeostasis in ischemia-reperfusion injury of the canine liver. J Surg Res. 1996;60:142-6.

15. Puglisi RN, Strande L, Santos M, Scheulte G, Hewitt CW, Whalen TV. Beneficial effects of rapamycin and cyclosporine in small bowel ischemia-reperfusion injury. J Surg Res. 1996;65:115-18.

16. Weinbrenner C, Liu GS, Downey JM, Cohen MV. Cyclosporine A limits myocardial infarct size even when administered after onset of ischemia. Cardiovasc Res. 1998;38:678-84.

17. Furuichi Y, Katsuta K, Maeda M, Ueyama N, Moriguchi A, Matsuoka $\mathrm{N}$, et al. Neuroprotective action of tacrolimus (FK506) in focal and global cerebral ischemia in rodents: dose dependency, therapeutic time window and long-term efficacy. Brain Res. 2003;965:137-45.

18. Naidu BV, Krishnadasan B, Farivar AS, Woolley SM, Thomas R, Van Rooijen N, et al. Early activation of the alveolar macrophage is critical to the development of lung ischemia reperfusion injury. $J$ Thorac Cardiovasc Surg. 2003;126:200-7.

19. Naidu BV, Woolley SM, Farivar AS, Thomas R, Fraga CH, Goss CH, et al. Early TNF- $\alpha$ release from the pulmonary macrophage in lung ischemia reperfusion injury. J Thorac Cardiovasc Surg. 2004; in press.

20. Mulligan MS, Vaporciyan AA, Warner RL, Jones ML, Foreman KE, Miyasaka M, et al. Compartmentalized roles for leukocytic adhesion molecules in lung inflammatory injury. J Immunol. 1995;154:1350-63.

21. St Peter SD, Moss AA, Mulligan DC. Effects of tacrolimus on ischemia-reperfusion injury. Liver Transpl. 2003;9:105-16.

22. Wakabayashi H, Karasawa Y, Tanaka S, Kokudo Y, Maeba T. The effect of FK506 on warm ischemia and reperfusion injury in the rat liver. Surg Today. 1994;24:994-1002.

23. Griffiths EJ, Halestrap AP. Protection by cyclosporin A of ischemia/ reperfusion induced damage in isolated rat hearts. $\mathrm{J} \mathrm{Mol} \mathrm{Cell} \mathrm{Cardiol.}$ 1993;25:1461-9.

24. Fiser SM, Tribble CG, Long SM, Kaza AK, Cope JT, Laubach VE, et al. Lung transplant reperfusion injury involves pulmonary macrophages and circulating leukocytes in a biphasic response. $J$ Thorac Cardiovasc Surg. 2001;121:1069-75.

\section{Discussion}

Dr Robert J. Keenan (Pittsburgh, Pa). I enjoyed your presentation. I have a couple of questions. Obviously I/R injury and immunomodulation go hand-in-hand, and it may be important specifically when looking at procurement of the donors to consider both factors in solutions derived at trying to improve overall lung function. I noted that your analysis of NF- $\kappa \mathrm{B}$ showed that the decline after 15 minutes was not in fact significant. Is your choice of using 15 minutes signifying the early phase when you expect $\mathrm{NF}-\kappa \mathrm{B}$ levels to be highest? Is that just not the right time? Maybe the NF- $\kappa \mathrm{B}$ is in fact not the right transcription factor, and maybe there is another one that is as important, if not more important, or maybe we're just not talking about the right effector cell.

Dr Woolley. Actually it is significant, but it isn't quantified in the article because we don't normally quantify our EMSAs. So it was just a qualitative decrease described with TAC treatment. If you actually go back and perform densitometry on all of the images and then derive statistics, it is a statistically significant decrease.

With respect to whether we're looking at the right time, all of the previous studies that have been performed in this model have shown that NF- $\kappa \mathrm{B}$ activation is maximal at 15 minutes of reperfusion. We believe that the 15-minute injury peak that occurs in this biphasic model is actually an early signaling event, because if you look at permeability indices or MPO concentrations, they peak at 15 minutes and then they go back down almost to baseline by approximately 1 to 2 hours, and then you go on to get your maximal full-blown injury at 4 hours of reperfusion. So we believe that this first event is actually a signaling event and that NF- $\kappa \mathrm{B}$ at 
this time is actually causing other cells, such as rat pulmonary endothelial cells and type II pneumocytes, to release more proinflammatory mediators that then set up the later full-blown injury.

As for whether we're looking at the right effector cell, and I presume you mean alveolar macrophages, we have performed several studies looking at alveolar macrophages. We have used gadolinium to suppress macrophage function, and we have performed macrophage depletion studies using clodronate liposomes. We found that depleting alveolar macrophages markedly decreases lung I/R injury. We have also repeated all of these studies in vitro using cultures of alveolar macrophages, type II pneumocytes, and rat pulmonary endothelial cells. We found that it is the alveolar macrophage that responds most to calcineurin inhibitors. Type II pneumocytes don't seem to do very much at all, and there is some response in rat pulmonary endothelial cells, but it's certainly not as great as the response you get in the macrophage. So I think we are actually looking at the right cells. As I say, when we go back and look at the densitometry, it is actually significant. But we don't normally quantify our EMSAs—we usually just make a qualitative analysis of them.

Dr Keenan. As a segue to the last point, then, does your BAL cell count analysis corroborate your statement that the alveolar macrophages are the ones that are diminished? When you look at the cell counts, you're looking potentially at PMNs as well as macrophages?

Dr Woolley. Yes. The cell counts are at 4 hours, so they are representing the late injury, and what we're saying is that the alveolar macrophages mediate the early 15 -minute time point, not the 4-hour injury. The 4-hour injury is all the neutrophils that have been sequestered, and at that point, yes, there is a whole milieu of cells with multiple cell types. We're only saying that the alveolar macrophages mediate the early time point, not the late time point as well, and that they set up the cascade that leads to the late injury.

Dr Keenan. The major question I have is related to the other effects that you talked about earlier. You honed in on the nuclear transcription factors, but those other issues, maybe not so much the mitochondrial factors, but the microcirculation factors, would seem to be important in the lung. If you gave this intravenously to the donor preoperatively, you might be able to achieve both some alveolar effects and microcirculatory effects.

Dr Woolley. Yes, and we have actually given intravenous TAC, as I said initially, and you're right, intravenous TAC has been shown to be markedly protective in $I / R$ in the liver and kidney. Yes, you may derive extra benefit from giving it like that. In this study we were trying to look at whether TAC could be potentially delivered through a nebulized route into the lungs of donors and minimize the systemic levels, because the other side of the coin is that if you have very high systemic concentrations, you may run into problems with nephrotoxicity or similar effects, and you don't want to jeopardize any of the other organs. However, I do agree with you that if it was at a low enough dose, you may well find that it is potentially beneficial and that it might protect the other organs being harvested at the same time.

Dr Thomas M. Egan (Chapel Hill, NC). Could you be a little more specific about exactly how you delivered the $200 \mu \mathrm{g}$ into the airway? Was it nebulized or did you just squirt it in? How did you get it in there?
Dr Woolley. It wasn't nebulized. We initially wanted to give it nebulized but had trouble finding a system that could do that. So we experimented with a lot of different ways of administering it to actually get it down into the lungs. Eventually what we came up with was that if we advanced the endotracheal tube right down onto the bifurcation and delivered it with the animal in a left lateral decubitus position, gravity would take it down into the left lung as well. We then injected $200 \mu \mathrm{L}$ with $300 \mu \mathrm{L}$ of air behind it to clear the dead space in the tube, so it would actually go down into the lungs. We did some radiolabeling studies and found that we were getting a ratio of 2.5:1, left to right, so most of the drug was getting into the left lung rather than the right, but we couldn't selectively intubate, so we couldn't inject it into the left lung alone.

Dr Egan. Have you performed any studies to look at providing this agent at reperfusion rather than 1 hour before? This could be a practical approach to treating non-heart-beating donors in whom you have an airway.

Dr Woolley. Only intravenously. We haven't done that yet with the endotracheal route. That's something we may think about doing. But so far we've only done it with the intravenous route, and it does provide protection if given at ischemia intravenously but not quite as great as when given as a pretreatment.

Dr Stephen D. Cassivi (Rochester, Minn). I am very interested in your work on this subject and I congratulate you for it.

I have a suggestion regarding possible future studies. I suppose the next step I would envision is to see whether this actually works in a simulated transplant situation. I know you're probably guided in Seattle by the fact that you also are very interested in I/R from the standpoint of the pulmonary artery hypertensive work that Dr Mulligan does. However, for those of us in the lung transplant field it would be interesting to see whether your findings carry over in an actual organ transplant model.

Dr Woolley. Yes, we're trying to work toward that. At the moment we don't have an orthotopic lung transplant model, but Dr Mulligan is currently doing some work with an orthotopic model in dogs, and if we can, we will try and use this with that model. At the moment we don't physically have the facilities to do that yet.

Dr Cassivi. You could start with the very simple rat single-lung transplant model. It's a relatively smaller model and fairly simple to get under way.

Dr Woolley. We are planning to use an orthotopic model in rats, but we do not have that up and running at the moment.

Dr Robert J. Korst (New York, NY). What do you think the downside of inhibiting NF- $\kappa \mathrm{B}$ in the airway is going to be in the donor? Have you thought about any potential downsides? NF- $\kappa \mathrm{B}$ is such a ubiquitous transcription factor, and there is undoubtedly some inflammation that's needed for such things like wound healing and so forth. Do you have any thoughts on that?

Dr Woolley. If you're giving it to the donor, then it shouldn't have much of an effect, because if you're giving it either 1 hour before harvesting the organs or at the time that you cause ischemia, you won't have time to worry about wound healing or similar problems in the donor. In the recipient, obviously that would be an issue, but it's going to be such a small dose when you've transplanted the lung into the recipient that it really shouldn't have much of an effect. 